\title{
RDUS
}

Revue de DROIT

UNIVERSITÉ DE SHERBROOKE

Titre : $\quad$ RESPONSABILITÉ DU MÉDECIN POUR UN DIAGNOSTIC ERRONÉ :

COMMENTAIRE DE L'AFFAIRE ॥ MASSINON ॥ C. ॥ GHYS ॥

Auteur(s) : $\quad$ François TÔTH

Revue : $\quad$ RDUS, 1996-1997, volume 27, numéro 1-2

Pages: $\quad 309-318$

ISSN : $\quad 0317-9656$

Éditeur : $\quad$ Université de Sherbrooke. Faculté de droit.

URI : $\quad$ http://hdl.handle.net/11143/12852

DOI : $\quad$ https://doi.org/10.17118/11143/12852 
Page vide laissée intentionnellement. 


\section{RESPONSABILITÉ DU MÉDECIN POUR UN DIAGNOSTIC ERRONÉ : COMMENTAIRE DE L'AFFAIRE MASSINON C. GHYS}

par François TÔTH*

Lorsqu'un médecin entreprend de soigner un patient, il doit mettre en oeuvre les moyens nécessaires, raisonnablement disponibles ${ }^{1}$ et conformes aux données actuelles de la médecine moderne. S'il ne le fait pas et qu'il rate son diagnostic, il ne pourra pas plaider «erreur de jugement». Il s'agit alors d'une faute professionnelle qui peut engager sa responsabilité.

C'est ce qu'a rappelé récemment la Cour supérieure dans l'affaire Massinon c. Ghys, ${ }^{2}$ une triste histoire de cancer du sein qui n'a pas été diagnostiqué à temps par le Dr Ghys. L'aspect le plus particulier de cette affaire, c'est qu'il est impossible d'affirmer avec certitude que le retard dans le diagnostic ait pu modifier l'évolution sournoise de la maladie et de ses séquelles. La Cour n'en a pas moins accueilli l'action.

Le 14 décembre 1987, Mme Massinon apprend, suite à une biopsie chirurgicale, qu'elle souffre d'un cancer au sein gauche alors que son médecin, le Dr Ghys, lui disait, depuis plusieurs mois et à la suite de moult examens, de ne pas s'inquiéter. Au moment du procès, Mme Massinon est atteinte de métastases osseuses. Elle souffre d'un cancer de stade quatre. ${ }^{3}$ Elle est incurable.

\section{Les fautes}

En 1987, Mme Massinon, une personne avertie et soucieuse de sa santé, consulte un «spécialiste du sein», le Dr Ghys. Ce dernier se spécialise depuis les

\footnotetext{
*. Avocat et professeur à la Faculté de droit de l'Université de Sherbrooke.

1. $\quad$ Chouinard c. Landry, [1987] R.J.Q. 1954 (C.A.).

2. [1996] R.J.Q. 2258.

3. Sur une échelle de 4 . Le stade 4 est terminal.
} 
années soixante dans le diagnostic et le traitement des maladies du sein. Il utilise plusieurs techniques diagnostiques dont la thermographie ${ }^{4}$ et la diaphanoscopie, ${ }^{5}$ techniques qualifiées par certains experts d'expérimentales. Le Dr Ghys utilise aussi les techniques reconnues de dépistage tels l'examen clinique et la mammographie. Fait non négligeable, le Dr Ghys a publié des ouvrages de vulgarisation destinés au grand public où il est fait mention de la nécessité d'un dépistage précoce du cancer dans le processus de rémission et quant aux chances de survie.

Mme Massinon a consulté le Dr Ghys à trois reprises en 1987. Jamais le Dr Ghys n'a posé de diagnostic de cancer.

La première consultation a lieu en janvier 1987. Mme Massinon, une jeune femme de 37 ans, était bien au fait de l'utilité de subir une mammographie pour une femme de son groupe d'âge. Inquiète de la texture et de l'état de ses seins à la suite de l'allaitement de son enfant, elle voulait en avoir le coeur net. Le Dr Ghys soumet la patiente à divers examens dont la diaphanoscopie et la thermographie. Le Dr Ghys indique à la patiente cet examen est normal, sans remarques particulières. Pourtant, les notes du Dr Ghys font état d'un diagnostic de dysplasie diffuse bilatérale 6 et qu'un suivi est souhaitable. Il lui indique de prendre rendez-vous dans un an.

C'est à la suite d'un voyage en Europe à l'été 1987 que Mme Massinon découvre un minuscule nodule à son sein gauche qui l'amène à reconsulter le Dr Ghys le 30 septembre 1987. Celui-ci la reçoit et la soumet à divers examens dont, à nouveau, la thermographie et la diaphanoscopie. Il ne procède qu'à une seule mammographie du sein gauche et ce, dans une seule incidence («angle»), ce qui est contraire, selon la preuve prépondérante, aux règles de l'art. Malgré des indications inquiétantes de cancer (au sein gauche) qui auraient nécessité des

4. Technique qui permet d'évaluer la température du corps et qui permettrait de découvrir une vascularisation inhabituelle à un endroit donné, ce qui serait un indice d'une lésion.

5. Étude de la densité du sein à l'aide de rayons infrarouges. Par translucidité, le médecin pourrait apercevoir des lésions sous forme d'ombres chinoises.

6. Selon le dictionnaire Robert, la dysplasie est une «anomalie dans le développement biologique (de tissu, d'organes, d'organismes) se traduisant par des malformations». 
$\begin{array}{cc}\text { (1996-97) } 27 \text { R.D.U.S. } & \text { Responsabilité du médecin } \\ \text { pour un diagnostic erroné : } & \text { commentaire de l'affaire Massinon c. Ghys }\end{array}$

investigations plus poussées, le Dr Ghys conclut à la normalité de l'examen. Bien qu'il veuille revoir la patiente dans trois mois, rien n'est dit à la patiente.

En novembre 1987, Mme Massinon note un changement à son sein gauche, ce qui l'affole. Elle obtient rapidement un rendez-vous avec le Dr Ghys le 4 décembre 1987. Les examens pratiqués ne sont pas normaux. Des images «suspectes» de malignité au sein gauche sont remarquées. Le Tribunal retient de la preuve que l'urgence de subir une biopsie chirurgicale a été niée par le médecin qui s'est fait plutôt rassurant. Tant et si bien que c'est à la suite de démarches de la patiente et de son mari qu'elle finira par obtenir un rendez-vous avec un chirurgien-oncologue le 8 décembre. Ce dernier fixe immédiatement une biopsie chirurgicale pour le 14 décembre 1987. Le chirurgien retire alors une masse de 5 x $2,5 \mathrm{~cm}$. Verdict : c'est le cancer.

Comment se fait-il que le Dr Ghys ait conclu à un état normal le 30 septembre 1987 et qu'il n'ait pas alors conseillé à la patiente de subir une biopsie chirurgicale? Y a-t-il eu faute médicale? En droit, c'est une question fort délicate car elle suppose l'analyse de la conduite professionnelle du médecin et, plus particulièrement, de l'élaboration de son diagnostic. Comme on le sait, le médecin n'est généralement tenu, comme l'avocat d'ailleurs, qu'à une obligation de moyens. Cela signifie qu' on ne jugera pas sa conduite à la lumière du résultat mais bien quant au respect des bons usages médicaux. Si ces bons usages médicaux sont respectés, il est admis que le médecin peut se tromper dans l'exercice de son jugement professionnel et qu'on ne pourra pas lui tenir rigueur de son erreur. C'est le plaidoyer de l'erreur de jugement non fautive. ${ }^{7}$

Pour le Dr Ghys, l'appréciation professionnelle de l'état de la patiente le 30 septembre 1987 indiquait un état bénin qui ne nécessitait pas d'investigations plus poussées. Dans la mesure où l'élaboration de ce diagnostic est conforme à la bonne pratique médicale, on ne peut remettre en cause sa valeur, surtout avec le bénéfice du «rétroscope» c'est-à-dire le bénéfice de la biopsie du 14 décembre 1987 qui démontrait le cancer, élément dont, bien sûr, le Dr Ghys ne disposait pas le 30 septembre.

7. Pour une critique de cette expression : J.-L. Baudouin, La responsabilité civile, $4^{\mathrm{e}}$ éd., Cowansville, Yvon Blais, 1994 au no 1208. 
Pour réussir dans une action en responsabilité contre un médecin où il est question de diagnostic erroné, il n'est pas suffisant de démontrer que le diagnostic n'est pas exact. Il faut aller plus loin et démontrer que l'erreur de diagnostic est le résultat d'une faute, soit d'un processus diagnostique non rigoureux, bâclé ou contraire aux données de la médecine moderne. À ce moment, l'erreur qui découle d'un processus fautif n'est plus à l'abri de la critique : il n'est que le résultat prévisible de la faute. ${ }^{8}$

L'élaboration du diagnostic doit, selon l'article 2.03.18 du Code de déontologie des médecins (c. M-9, r.4), se faire avec la plus grande attention, en utilisant les méthodes scientifiques les plus appropriées et, si nécessaire, en recourant aux conseils les plus éclairés. Or, le Dr Ghys a fait l'objet d'une plainte déposée par le syndic du Collège des médecins, lui reprochant un manquement à cette obligation dans le traitement de Mme Massinon. Le Dr Ghys a plaidé coupable à la plainte. Très habilement, la demande a voulu utiliser cet aveu de culpabilité, non pas comme aveu extrajudiciaire dans l'instance civile établissant la véracité des faits reprochés (ce qui est incontestablement possible à certaines conditions ${ }^{9}$ ), mais comme déclaration antérieure contradictoire minant la crédibilité du témoin quant à la suffisance des examens médicaux (art. 2871 C.c.Q.). Le Tribunal a permis cette preuve.

Le médecin, membre du Collège des médecins, adhère de ce fait à la médecin moderne. Il doit exercer sa profession selon des principes scientifiques (art. 2.03.14, Code de déontologie des médecins). S'il utilise des méthodes non éprouvées scientifiquement, il peut commettre non seulement une faute déontologique mais également une faute civile à l'égard de son patient en négligeant de lui fournir des soins reconnus et adéquats. ${ }^{10}$ Certaines des

8. Voir Cloutier c. Ahad (2 juin 1980), Montréal 500-05-003-404-751, J.E. 80-514 (C.S.) pour une revue complète des principes en matière de responsabilité médicale pour un diagnostic erroné. Voir aussi A. Bernardot et $\mathrm{R}$. P. Kouri, La responsabilité civile médicale, Sherbrooke, Éditions Revue de droit Université de Sherbrooke, 1980 aux pp. 196 et s.; P. Lesage-Jarjoura, J. Lessard et S. Philips-Nootens, Éléments de responsabilité civile médicale : le droit dans le quotidien de la médecine, Cowansville, Yvon Blais, 1995 aux pp. 211 et ss.

9. Voir J.-L. Baudouin, supra note 7 au no 47.

10. Les aléas des techniques diagnostiques non éprouvées devraient également être divulgués au patient dans le cadre de l'obligation de renseignement du médecin. 
méthodes utilisées par le Dr Ghys (thermographie, diaphanoscopie) ne sont pas reconnues par la médecine et on ne pouvait y accorder une valeur diagnostique, surtout à la lumière des résultats négatifs de ces examens. En fait, cela ne démontrait rien. Le médecin qui fonde alors son diagnostic sur des méthodes non reconnues ne peut pas plaider simple erreur de jugement ou liberté thérapeutique.

La preuve experte permet également de reprocher au Dr Ghys d'avoir négligé ce qu'un bon médecin prudent et diligent n'aurait pas manqué de noter, notamment quant aux signes cliniques de cancer chez Mme Massinon ainsi que d'avoir procédé, le 30 septembre 1987, à l'examen clinique d'une façon inappropriée (en position debout). L'erreur de diagnostic est ici le résultat d'un procédé diagnostique inadéquat. La faute (méthode diagnostique incorrecte) ne peut alors devenir un moyen de défense (aléa inhérent à l'art médical).

Une autre faute qui explique l'erreur de diagnostic est d'avoir procédé à une seule mammographie dans une seule incidence. La médecine enseigne qu'il faut plutôt procéder à une mammographie bilatérale (c'est-à-dire des deux seins) dans deux incidences. L'examen était donc incomplet. Les risques d'erreur augmentaient...

Au surplus, les indices révélés par les examens, même incomplets, du Dr Ghys auraient dû suffire à conclure à la malignité possible de la tumeur observée. Il aurait dû alors en aviser sa patiente dès le 30 septembre 1987 et assurer le suivi nécessaire (soit poursuivre l'investigation rapidement par une biopsie chirurgicale). Même l'examen du 4 décembre 1987 est incomplet, et la réaction du médecin inappropriée devant l'urgence de la situation.

Remarquons que le Tribunal peut décider de la justesse d'un diagnostic sans usurper sa fonction, ni prétendre trancher une controverse scientifique. Appelé à juger un litige sur la base des faits mis en preuve et des opinions des experts qui ont déposé devant lui, le Tribunal peut préférer l'opinion d'un expert à celle d'un autre. ${ }^{11}$ Il pouvait décider que, lors de la consultation du 30 septembre 1987, des signes inquiétants de cancer existaient et que le Dr Ghys 
aurait dû agir autrement. Le Tribunal ne fait pas alors office de «Sorbonne médicale» en matière de traitement médical mais décide de la conformité du comportement professionnel du médecin en l'espèce avec la bonne pratique médicale d'alors.

\section{Le dommage et la causalité}

La faute dans l'élaboration du diagnostic engendre-t-elle la responsabilité civile du Dr Ghys? Il faut maintenant se demander si la faute a causé un dommage. Plus exactement, il faut déterminer, à la lumière des enseignements de la Cour suprême, quel est exactement le dommage que cette faute a causé. ${ }^{12}$

La faute du Dr Ghys a retardé la découverte de la maladie de $\mathbf{7 5}$ jours et, par voie de conséquence, son traitement. Évidemment, la faute médicale n'est pas la cause du cancer, mais peut-on dire qu'elle a privé Mme Massinon de la possibilité de le vaincre? Autrement dit, le médecin peut-il être responsable d'avoir privé sa patiente d'une chance de guérir? La difficulté, en l'espèce, c'est qu'on est incapable de dire, du point de vue scientifique, que le retard dans le traitement aurait pu vraiment changer l'évolution de la maladie.

La Cour suprême, dans un jugement très étoffé, ${ }^{13}$ a écarté la perte de chance de guérir comme fondement à une action en responsabilité civile si l'on est incapable d'établir le lien de causalité entre la faute et le dommage. En effet, ou bien la faute médicale cause le dommage, ou bien elle est neutre dans l'évolution de la maladie. Si la faute médicale cause le dommage, alors le patient sera indemnisé pour tous les dommages que la faute a causés. ${ }^{14} \mathrm{Si}$ la faute médicale n'a pas joué de rôle dans le développement ou l'aggravation de la maladie, alors le médecin ne peut pas être condamné pour un dommage qu'il n'a pas causé.

12. Laferrière c. Lawson, [1991] 1 R.C.S. 541.

13. Ibid.

14. Il pourrait s'agir aussi de l'aggravation d'un état préexistant. 
Ce raisonnement est valable si l'on ne considère que le dommage final (la mort, le handicap). Mais, si l'on est incapable d'établir le lien de causalité entre la faute et le préjudice final, cela ne signifie pas que la faute n'a pas causé d'autres dommages, par exemple les souffrances morales endurées par le patient qui débute, déjà avec quelques longueurs de retard, un combat contre le cancer...

C'est exactement ce dont il s'agit dans l'affaire Massinon c. Ghys. La patiente réclame une somme de $60000 \$$ pour perte de jouissance de la vie, angoisse et stress continuels face aux perspectives d'aggravation de son état et d'abrégement de la vie. ${ }^{15}$ L'on ne réclame rien pour le préjudice physique ou économique rattaché au délai dans l'établissement du diagnostic. La Cour suprême ${ }^{16}$ avait accordé, en 1991 et dans des circonstances semblables, $17500 \$$ à titre de dommages moraux pour une patiente qui a vécu de telles angoisses pendant près de trois ans depuis la découverte du cancer (et de la faute médicale) en mars 1975 jusqu'à son décès, le 27 janvier 1978. En l'espèce, Mme Massinon vit, et vit toujours son drame depuis $1987 . .$.

La première difficulté consiste à isoler les conséquences de la faute des conséquences de la maladie elle-même. En effet, l'angoisse reliée à la divulgation de la maladie mortelle n'est pas liée à la faute médicale. Par contre, l'angoisse supplémentaire causée par le retard dans le traitement l'est. Une tâche difficile attend le Tribunal.

La demande a présenté une preuve fort éloquente de la détresse psychologique très grave causée à la patiente par la faute médicale : dépressions nerveuses, perte de l'espoir et du goût de vivre, tentatives de suicide ${ }^{17}$...Tout cela est exacerbé par le message transmis aux femmes par la communauté médicale dont le Dr Ghys s'est fait le porte-parole : le dépistage précoce permet un bon

15. Quant à ce dernier aspect (abrégement de la vie comme dommage objectif), la décision de la Cour suprême dans l'affaire Augustus c. Gosset, [1996] 3 R.C.S. 268 exclut toute possibilité à cet égard.

16. Supra note 12 .

17. À ce sujet, il faut lire la décision de la Cour. On y décrit avec beaucoup de sensibilité tout le calvaire vécu par Mme Massinon. On consultera également en annexe II du jugement, le résumé du dossier médical de la demanderesse. 
pronostic et des traitements moins mutilants. Pour Mme Massinon, tout cela est désormais réduit à néant : elle n'a pas été «prise à temps» et cela est dû à la faute du médecin. Il s'agit là d'une souffrance additionnelle à la souffrance normalement liée au cancer. On ne peut pas dire que la réaction de Mme Massinon soit excessive dans les circonstances. On ne peut pas dire non plus qu'elle ait tort de croire qu'un dépistage précoce aurait pu changer les choses : toute la littérature scientifique est dans ce sens.

De plus, le niveau d'évolution de la maladie peut modifier la thérapie. Si la maladie de Mme Massinon avait pu être diagnostiquée plus tôt à un stade moins avancé, le traitement aurait pu être moins pénible. Mais quel était le stade du cancer de Mme Massinon en septembre 1987? Voilà une deuxième difficulté. En effet, on ne sait pas si, objectivement, la maladie de la demanderesse a évolué de façon sensible pendant ces 75 jours : les examens effectués par le Dr Ghys et les données notées au dossier sont incomplets. L'impossibilité dans laquelle se trouve maintenant la demanderesse de faire la preuve de l'évolution de sa maladie résulte de la faute du médecin. À ce moment, il est permis de faire supporter au médecin le fardeau de prouver que la maladie n'a pas évolué (donc que sa faute a été neutre) plutôt que d'imposer à la demanderesse de prouver le contraire. En violant son obligation de rendre compte au patient de toutes les données pertinentes à sa condition, le médecin assume le fardeau de démontrer leur absence de lien causal. ${ }^{18}$ Le droit est sage : une faute ne doit pas devenir un moyen de défense.

Le Tribunal accorde entièrement la somme réclamée : 60 000\$.

\section{Observations}

L'affaire Massinon c.Ghys est un jugement important car il s'agit de l'une des premières affaires plaidées depuis la décision de la Cour suprême dans

18. Voir aussi Snell c. Farrell, [1990] 2 R.C.S. 311 , Houde c. Côté, [1987] R.J.Q. 723 (C.A.), Gburek c. Cohen, [1988] R.J.Q. 2424 (C.A.). Aussi, J.-P. Ménard, «La causalité dans les causes de responsabilité médicale depuis les affaires Lawson et Farrell» dans Développements récents en droit de la santé, vol. 24, Cowansville, Yvon Blais, 1991 à la p. 1. Pour une application récente : Stéfanik c. Hôpital Hôtel-Dieu de Lévis, [1997] R.J.Q. 1332 (C.S.). 
Laferrière c. Lawson. La somme, fort modeste, accordée dans ce dernier jugement (17 500\$) rendait ces cas difficiles et coûteux à plaider.

Bien que les conséquences particulières de la faute médicale pour Mme Massinon aient atteint un niveau qu'on pourrait qualifier d'exceptionnel, le jugement confirme qu'on ne doit plus considérer légèrement le dommage psychologique causé par la faute. La somme accordée, $60000 \$$, peut apparaître substantielle selon les paramètres jurisprudentiels habituels mais respecte le plafond de 100000 \$ établi en 1978 par le Cour suprême, ${ }^{19}$ maintenant d'environ $243000 \$ .^{20}$ À notre avis, la somme de $60000 \$$ (25\% du plafond ajusté) est pleinement justifiée dans les circonstances.

Depuis quelques années, l'on assiste à un développement jurisprudentiel intéressant en matière d'indemnisation du préjudice, ${ }^{21}$ particulièrement au niveau des dommages non pécuniaires, tant corporels que moraux. La protection accordée par le droit à la personne s'étend au-delà de sa sécurité purement physique. La personne est dorénavant vue dans son intégralité. ${ }^{22}$ Pensons aux affaires suivantes :

- Augustus c. Gosset ${ }^{23}$ : reconnaissance du solatium doloris comme dommage indemnisable;

- Québec (Curateur public) c. Syndicat national des employés de l'Hôpital St-Ferdinand ${ }^{24}$ : octroi de dommages non pécuniaires à des victimes handicapées mentales et rejet de la thèse fonctionnelle;

19. Andrews c. Grand and Toy Alberta Ltd., [1978] 2 R.C.S. 229. Ce plafond doit-il être revu? On a bien accordé $300000 \$$ à titre de dommages moraux pour diffamation : Hill c. Église de scientologie de Toronto, [1995] 2 R.C.S.1130.

20. Augustus c. Gosset, supra note 15.

21. Sur la nouvelle classification tripartite du préjudice et ses difficultés : N. Vézina, «Préjudice matériel, corporel et moral : variations sur la classification tripartite du préjudice dans le nouveau droit de la responsabilité», (1993) 24 R.D.U.S. 161.

22. On doit parler d'intégrité corporelle dans son sens global, i.e. comme incluant l'intégrité physique et psychique. Voir N. Vézina, supra note 21 aux pages 182-183.

23. Supra note 15.

24. [1996] 3 R.C.S. 211. 
- $\quad$ Hill c. Église de scientologie de Toronto ${ }^{25}$ : octroi de $300000 \$$ comme dommages à la suite de diffamation;

- Éditions Vice-Versa inc. c. Aubry ${ }^{26}$ : condamnation à $2000 \$$ pour la publication sans autorisation dans un magazine de la photographie d'une personne assise dans un lieu public; violation du droit à la vie privée.

Plus que jamais, les avocats devront être vigilants. Il sera plus difficile de conseiller adéquatement un client lors de négociations. Les risques du procès seront désormais très réels même en l'absence de blessures physiques ou de dommages matériels. L'on ne pourra plus plaider facilement que «la faute n'a pas causé de dommage».

25. Supra note 19.

26. [1996] R.J.Q. 2137 (C.A.). Requête pour autorisation de pourvoi à la Cour suprême accueillie, greffe 25579, le 6 février 1997. 